\title{
SRI'S REAL-TIME SPOKEN LANGUAGE SYSTEM
}

\author{
Patti Price and Robert C. Moore
}

\author{
SRI International \\ Menlo Park, CA 94025
}

\section{PROJECT GOALS}

This project involves the integration of speech and naturallanguage processing for spoken language systems (SLS). The goal of this project, to develop a multi-modal interface to the Official Airline Guide database, is being developed along two overlapping research and development lines: one focussed on an SLS kernel for database query, and the other on the full interactive system.

\section{RECENT RESULTS}

- Speaker-dependent and speaker-independent demos to illustrate the combined recognition/natural language system and accompanying graphical user interfaces.

- Improved robustness through the development of a template matcher for generating database queries; the template matcher has a tunable parameter to control how much constraint is ignored, so that wrong answers can be traded with no answers.

- Implementation of a bottom-up parser for CLE-formalism grammars; the new parser is about twice as fast as our original left-corner parser, and about 17 times faster than an initial bottom-up parser.

- Exploration of two schemes to integrate the recognizer and current NL schemes: N-best recognition with a statistical grammar, and recognition guided by a probabilistic finitestate representation of the templates.

- Evaluation of SRI's NL, SLS, and speech recognition technologies. SRI's February- 91 weighted sentence error rate for ATIS Class A sentences was $33.8 \%(\mathrm{NL})$ and $44.1 \%$ (SLS); word error rate for the Resource Management speaker independent speech recognition evaluation was $17.6 \%$ with no grammar and $4.8 \%$ with the standard wordpair grammar.

- Improvements in the CLE grammar: extended coverage of numerical expressions, ATIS domain sortal restrictions, and conjoined noun phrases.

- Implementation of tied-mixture hidden Markov models, which resulted in a $20 \%$ reduction in the word-error rate compared to the discrete-density version.
- Training of a discrete-density DECIPHER using 20,000 sentences of read and spontaneous speech from Resource Management, TIMIT, and ATIS corpora. We achieved 10\% word error on the June-90 ATIS test set.

- New techniques for statistical language models: a back-off estimation algorithm, Good-Turing estimates, and interpolation of word-based grammars with class-based grammars. Current test-set perplexity ranges from 15 to 30 .

- Initial implementation of fast-search recognition algorithms for near real-time recognition.

- Initial implementation of speaker-adaptation using tiedmixture codebook adaptation.

- Implementation of an HMM reject-word model for dealing with noises and out-of-vocabulary items in digit recognition tasks; we plan to incorporate this in our ATIS SLS.

\section{PLANS FOR THE COMING YEAR}

- Continue data collection and analysis and expand the ATIS domain.

- Implement a new architecture to combine the advantages of the robust template matcher with the new bottom-up parser. The parser will feed individual constituents to the template matcher, even if it cannot find a complete interpretation of an utterance, and will also form the basis of a phrase-level statistical language model to guide recognition (see Jackson et al., this volume).

- Continue efforts in statistical language modeling: Determine why low perplexity systems do not always improve accuracy; Develop means for making efficient use of language-model training data, and for using out-of-task training data; Combine statistical language modeling with linguistic models of syntax and semantics.

- Improve acoustic modeling performance: consistency modeling, speaker-adaptation, rejection modeling (including altemative topologies and training techniques), spontaneous speech phenomena (variations in phonology and speaking rate), corrective training; Implement a continuous-distribution version of DECIPHER. 